\title{
Review
}

\section{Central Neural Mechanisms Involved in the Control of Jaw Movement during Postnatal Development}

\author{
Shiro NaKamura ${ }^{* 1)}$, Shoko Nagata ${ }^{2)}$, Mutsumi NonAKA ${ }^{3)}$, \\ Akiko Nishimura ${ }^{3)}$, Kouta NagoYA ${ }^{4)}$, Masanori DanTSUJi ${ }^{5)}$, \\ Kiyomi NaKaYama $^{1)}$, Ayako Mochizuki ${ }^{1)}$, Takehiko IJImA ${ }^{3)}$, \\ Masahiko Ozeki ${ }^{5)}$, Matsuo YAmamoto ${ }^{2)}$ and Tomio Inoue $^{1)}$
}

\begin{abstract}
Mechanisms responsible for controlling masticatory muscle activity during suckling and mastication initiate in the brain. Premotor neurons situated in the brainstem transmit masticatory motor commands to the jaw-closing and jaw-opening motoneurons, which in turn activate the masticatory muscles. The premotor neurons receive inputs from multiple sources such as the orofacial sensory organs, the cerebral cortex, and the central pattern generator in the brainstem. These neural circuits might be important for controlling jaw movement, although their properties in this regard are likely to be altered in postnatal development as the oral motor behavior of mammals shifts significantly from suckling to chewing during the early postnatal period. This timing of jaw movement development mimics that of the orofacial musculoskeletal apparatus. The current article reviews findings on local neural circuits of trigeminal motoneurons and premotor neurons, during early development in rats. We describe findings from electrophysiological recordings, optical imaging with voltage-sensitive dyes, calcium imaging, and laser photolysis of caged glutamate. We also review research into the subtypes of premotor neurons in the supratrigeminal region (SupV) that differ in their electrophysiological properties and possess different morphological characteristics. We further describe how trigeminal motoneurons receive glutamatergic, glycinergic, and GABAergic inputs from the SupV and the reticular formation dorsal to the facial nucleus. Finally, we discuss how these synaptic and intrinsic membrane properties change in postnatal development. Taken together, the findings reviewed herein suggest that these neural circuits are important in diverse oral motor behaviors, and that their postnatal changes are involved in the transition from suckling to mastication functions.
\end{abstract}

Key words : neural mechanisms, jaw movement, premotor neurons, postnatal development

\footnotetext{
1) Department of Oral Physiology, Showa University School of Dentistry, 1-5-8 Hatanodai, Shinagawa-ku, Tokyo 1428555, Japan.

2) Department of Periodontology, Showa University School of Dentistry.

3) Department of Perioperative Medicine, Division of Anesthesiology, Showa University School of Dentistry.

4) Department of Oral Biological Science, Division of Dysphagia Rehabilitation, Niigata University Graduate School of Medical and Dental Sciences.

5) Department of Implant Dentistry, Showa University School of Dentistry.

* To whom corresponding should be addressed.
} 


\section{Introduction}

Feeding-related motor activity such as suckling and mastication serves a vital function for the survival and development of mammals. Mechanisms responsible for controlling jaw movement during suckling and mastication exist in the brain ${ }^{1-4)}$, wherein the fundamental motor patterns for these behaviors originate in the central pattern generator (CPG) located in the brainstem. Here, they are modulated by sensory information on food properties and by descending inputs from the higher brain ${ }^{4,5)}$. These motor commands are transmitted to the trigeminal motoneurons (TMNs) that innervate the masticatory muscles via premotor neurons. Such premotor neurons are distributed in various brain regions including the lateral reticular formation of the lower brainstem in the pons and medulla oblongata ${ }^{6-18)}$. In addition, these premotor neurons also receive ascending and descending inputs from a number of regions: the orofacial structures ${ }^{6-8)}$, the masticatory $\mathrm{CPG}^{4,9)}$, the cerebral cortex ${ }^{10,11)}$, the hypothalamus ${ }^{12)}$, and the amygdala ${ }^{13)}$. This neural architecture indicates that the neural circuits including premotor neurons and motoneurons are involved in controlling masticatory muscle activities via ascending and descending inputs during jaw movement. The properties of these neural circuits differ among postnatal periods ${ }^{19)}$ because feeding behavior of mammals changes substantially from suckling to chewing during development, which is accompanied by the formation of the oral and facial musculoskeletal system during the early postnatal period.

In this review, we describe findings on the location, electrophysiology, and morphological characteristics of premotor neurons that target the TMNs. We further review properties of the related neural circuits, as identified by studies using rat in vitro slice preparations during early postnatal development.

\section{Location and properties of premotor neurons targeting the TMNs}

Retrograde axonal tracing studies have identified premotor neurons projecting to the trigeminal motor nucleus $(\mathrm{MoV})$ in the regions surrounding the MoV. These regions comprise the supratrigeminal region $(\mathrm{SupV})$, the intertrigeminal region $(\mathrm{IntV})$, the dorsal parts of the principal sensory trigeminal nucleus $(\operatorname{PrV})$, and the regions dorsal to the $\operatorname{PrV}(\mathrm{dRt})^{20-22)}$. We investigated the properties of synaptic inputs from these premotor neurons to the jaw-closing and jawopening motoneurons in coronal and horizontal brainstem slices from neonatal and juvenile rats [postnatal day (P) 1-12 $]^{23-25)}$. We used electrical stimulation and laser photolysis of caged glutamate with simultaneous whole-cell patch-clamp and optical voltage recording. Electrical stimulation and laser photolysis of caged glutamate in the SupV ${ }^{23)}$ and reticular formation dorsal to the facial nucleus $(\mathrm{RdVII})^{25)}$ elicits fast excitatory and inhibitory postsynaptic responses, mediated by glutamate, $\mathrm{GABA}_{\mathrm{A}}$, and glycine receptors in the jaw-closing masseter motoneurons (MMNs) and jaw-opening digastric motoneurons (DMNs). Furthermore, systematic photostimulation of multiple sites of the SupV, IntV, dorsal PrV, and dRt evokes postsynaptic currents (PSCs) in the majority of MMNs and DMNs ${ }^{24}$ (Fig. 1). Remarkably, the stimulation of the lateral SupV induces burst postsynaptic currents (PSCs) in MMNs more frequently than low-frequency PSCs 

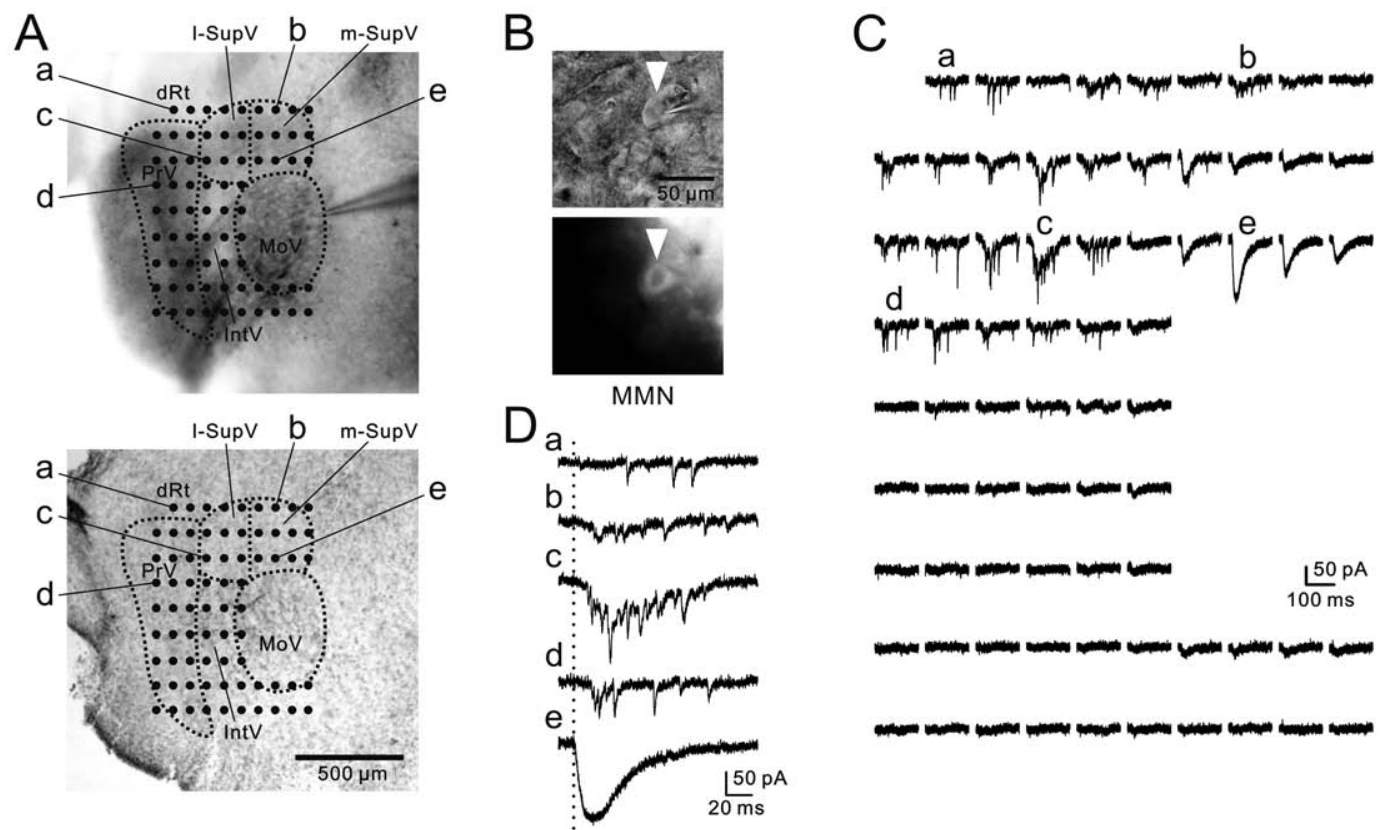

Fig. 1. Locations of the premotor neurons targeting the MMNs identified by laser photolysis of caged glutamate. (A) Representative video image (upper panel) and the corresponding Nissl-stained image (lower panel) of a transverse slice from a P4 rat overlaid with 73 stimulation sites. (B) Translucence (upper panel) and epifluorescence (lower panel) images of the recorded MMN (arrowhead). (C) Corresponding postsynaptic responses for the same stimulation sites as shown in (A). (a-e) denote postsynaptic currents evoked by stimulation applied to the positions indicated by (a-e) in (A). (D) The expanded traces of $(\mathrm{a}-\mathrm{e})$ in $(\mathrm{C})$. MMN, masseter motoneurons; P4, postnatal day 4 ; $\mathrm{MoV}$, trigeminal motor nucleus; $1-\mathrm{SupV}$ and $\mathrm{m}-\mathrm{SupV}$, lateral and medial supratrigeminal regions, respectively; IntV, intertrigeminal region; PrV, principal sensory trigeminal nucleus ; dRt, region dorsal to the PrV. Source: Reproduced from Nonaka et al (2012) ${ }^{24)}$ with permission from SAGE. Copyright ${ }^{\complement}$ 2012, SAGE Publications. All rights reserved.

in MMNs or burst PSCs in DMNs (Fig. 1C). These data suggest that the SupV contains premotor neurons sending both excitatory and inhibitory inputs to the MMNs and DMNs, and that the lateral SupV supplies burst inputs specifically to the neonatal MMNs.

The electrophysiological and morphological characteristics of SupV premotor neurons targeting the $\mathrm{MoV}$ were further characterized in neonatal rat brainstem slices, using whole-cell recording and calcium imaging of premotor neurons. These methods were combined with antidromical stimulation of the $\mathrm{MoV}$ in slices stained with calcium indicators, fura- $2 \mathrm{AM}$ and fluo- $8 \mathrm{AM}^{26)}$. Approximately $10 \%$ of neurons selected among the SupV showed a clear elevation of intracellular $\mathrm{Ca}^{2+}$ concentration in response to a brief electrical stimulation of the MoV. Subsequent whole-cell recordings confirmed that the majority of these neurons could follow a $100-\mathrm{Hz}$ triple shock of the MoV at constant latencies without action potential failure, even in the presence of antagonists for glutamate, $\mathrm{GABA}_{\mathrm{A}}$, and glycine receptors. These results indicate that the premotor neurons tested project to the MoV. Neurons differ in their steady-state firing, i.e., the average firing frequency over the last half of a 1-s depolarizing current pulse from the resting membrane potential. Two firing types of antidromically activated SupV premotor neurons were 
A

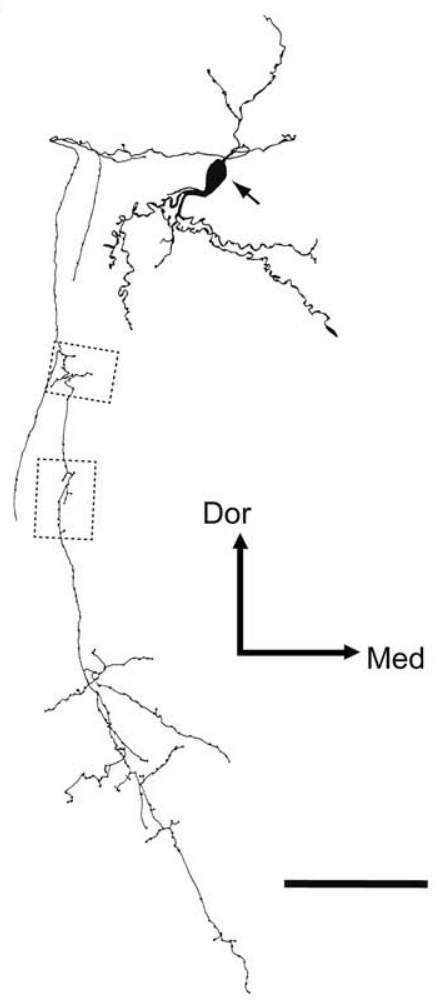

B

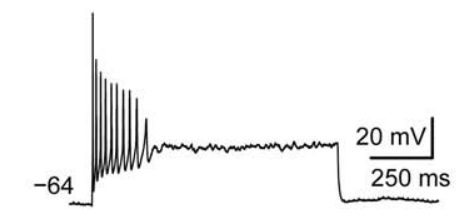

C

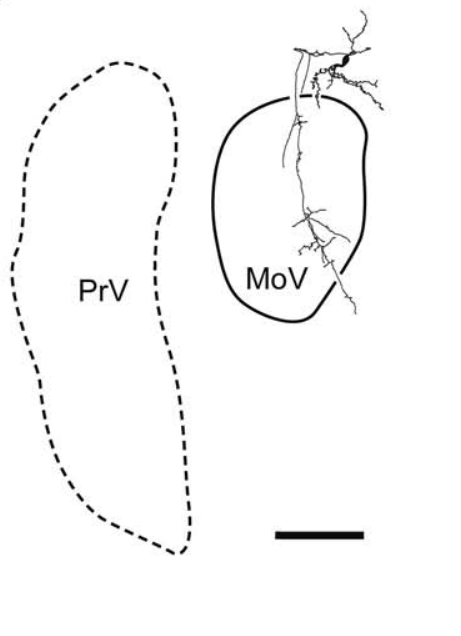

D

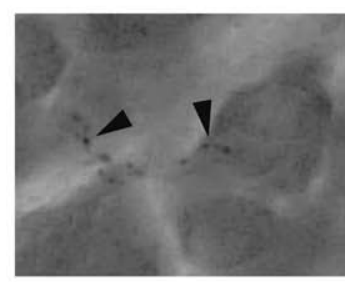

E

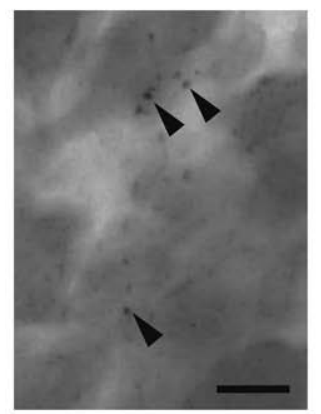

Fig. 2. Physiological and morphological properties of a SupV premotor neuron. (A) Camera lucida reconstruction of a biocytin-filled neuron showing the soma (arrow), axons, and dendrites. (B) Low-frequency firing characteristics of the neuron shown in (A). (C) Camera lucida reconstruction showing the location of this neuron in relation to the MoV and PrV. (D, E) Photographs of axonal fibers and terminals in the MoV indicated by dotted rectangles in (A). Arrowheads indicate the terminal arbors with terminal boutons. Synaptic contacts of this neuron are made on the soma and juxtasomatic region of trigeminal motoneurons. Dor, dorsal; Med, medial ; MoV, trigeminal motor nucleus; PrV, principal sensory trigeminal nucleus; SupV, supratrigeminal region. The scale bars are $100 \mu \mathrm{m}$ (A), $200 \mu \mathrm{m}$ (C), and $20 \mu \mathrm{m}$ (D, E). Modified from Nakamura et al $(2014)^{26)}$ with permission from APS. Copyright $\left.^{(}\right) 2014$, the American Physiological Society. All rights reserved.

identified based on their steady-state firing properties: neurons fire at a frequency either higher (HF) or lower (LF) than $33 \mathrm{~Hz}$. HF neurons have a narrower action potential and a larger after-hyperpolarization (AHP) amplitude than LF neurons. Morphologically, the axons of all HF neurons and half of the LF neurons project to the dorsomedial aspect of the MoV, while half of the LF neurons project to its dorsolateral aspect (Fig. 2). In addition, the dendrites of some HF neurons extend to the PrV, whereas the dendrites of all LF neurons are confined within the SupV. These findings suggested that the SupV premotor neurons projecting to the MoV show different electrophysiological properties and distinct dendritic and axonal morphologies, and that such heterogeneity might be involved in diverse oral motor behavior.

Recent observations in our laboratory suggested that a specific neuronal subpopulation in the SupV expresses the transcription factor Phox $2 b$, which plays an important role in development of the autonomic nervous system ${ }^{27}$. This subpopulation was identified in transgenic rats modified 
to express EYFP in Phox2b-positive neurons, which in the SupV clearly differ from the Phox2bnegative neurons in their electrophysiological properties. Approximately $50 \%$ of Phox $2 \mathrm{~b}$-positive neurons project to the $\mathrm{MoV}$, and it is possible that this neuronal group has a distinct role in oral motor functions such as suckling and/or mastication.

\section{Postnatal development of synaptic transmission from premotor neurons to TMNs}

In mammals, feeding behavior remarkably converts from suckling milk to chewing solid food during the early postnatal period. During this period, the activity of feeding-related muscles also changes from low to high corresponding to the properties of the feeding material ${ }^{1,2,28)}$. This suggests that motor commands to these masticatory muscles change in parallel with the postnatal development of the orofacial musculoskeletal structures. Since the TMNs provide the final motor output to jaw-closing and jaw-opening muscles ${ }^{29,30)}$, the common opinion in the field is that the input-output properties of these motoneurons change in line with the alteration of motor commands ${ }^{4,19,31)}$.

Previous laser photolysis of MNI-caged glutamate demonstrated that MMNs in P1-5 rats show glutamatergic-burst PSCs predominantly in response to SupV photostimulation in the presence and absence of $\mathrm{GABA}_{\mathrm{A}}$ and glycine receptor antagonists ${ }^{24)}$. In contrast, low-frequency PSCs are the major responses in both MMNs and DMNs to photostimulation of the SupV in P9-12 rats ${ }^{32)}$ (Fig. 3A) ; however, when higher concentrations of caged glutamate are applied to P9-12 rats, SupV photostimulation elicits burst PSCs in MMNs. These results indicate that the input-output gain of some SupV premotor neurons decreases during postnatal development.

Jaw-closing motoneurons have large and well-developed dendrites, which receive ascending and descending inputs ${ }^{33,34)}$. Thus, it is possible that dendritic properties of the jaw-closing motoneurons also change with increasing postnatal age, and we investigated this hypothesis during early development in rats ${ }^{35)}$. We recorded the glutamatergic responses evoked by local photostimula-

A

Glutamatergic inputs

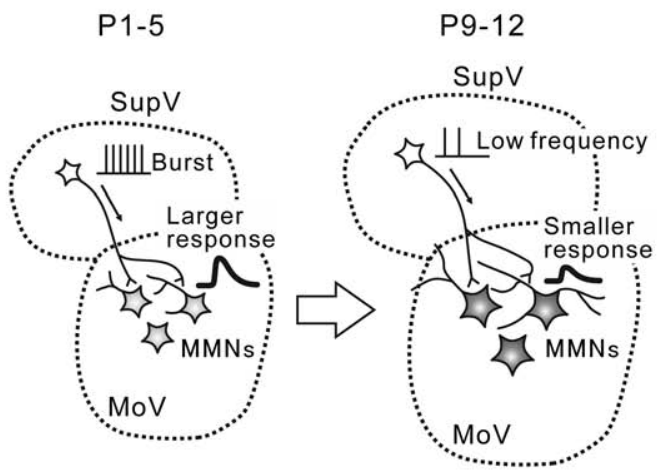

B GABAergic/glycinergic inputs

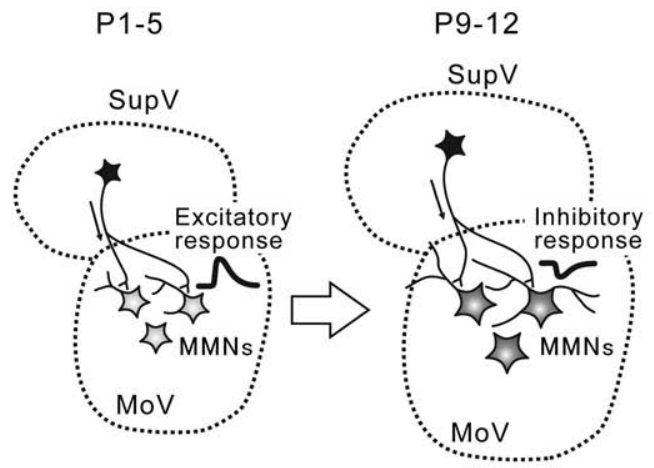

Fig. 3. Schematic illustration of premotor neuronal inputs to MMNs during early postnatal development. (A) Postnatal development of glutamatergic inputs to the MMNs. (B) Postnatal development of GABAergic and glycinergic inputs to the MMNs. MMNs, masseter motoneurons; MoV, trigeminal motor nucleus; SupV, supratrigeminal region. 
tion of the dendrites, using laser photolysis of caged glutamate combined with somatic whole-cell recordings. In MMNs of P2-5 rats, dendritic photostimulation evokes depolarizing glutamatergic responses in the presence of tetrodotoxin. With increasing stimulus intensity, $75 \%$ of $\mathrm{MMNs}$ show a step-like increase in depolarization that is suppressed by a NMDA receptor antagonist, indicating NMDA spikes/plateau potentials ${ }^{36,37)}$. However, the glutamatergic responses are markedly smaller in amplitude and shorter in duration in P9-12 than those measured in P2-5 rats, and few neurons exhibit NMDA spikes/plateau potentials in the MMNs of P9-12 rats. These data suggested that the glutamatergic input properties of jaw-closing motoneuron dendrites change during the first two postnatal weeks (Fig. 3A). We also recently found that dendritic glutamatergic responses are enhanced by the application of 5-HT in the MMNs of neonatal rats (unpublished observation). Thus, serotonergic modulation of dendritic properties might change during the early postnatal period.

Interestingly, whether inputs from SupV and RdVII to MMNs are excitatory or inhibitory depends on the developmental stage of the rat ${ }^{23,25)}$. P1-4 rats show excitatory effects via the activation of glutamate, glycine, and putative $\mathrm{GABA}_{\mathrm{A}}$ receptors, while P9-12 rats exhibit inhibitory effects after glycinergic and GABAergic input ${ }^{23}$ 25) (Fig. 3B). These data suggested that glutamatergic synaptic inputs from SupV to MMNs are excitatory throughout the postnatal period, while glycinergic and GABAergic inputs convert from excitatory to inhibitory at around P7.

\section{Postnatal development of intrinsic properties of the TMNs}

Intrinsic membrane properties of the jaw-closing motoneurons are altered together with changes to their input properties. Yamaoka et al $^{38)}$ reported that the resting membrane potentials in rat MMNs are relatively constant throughout postnatal development (P4-27), but that the input resistance progressively decreases over the same period, and although the amplitude of the action potential does not change, its half duration decreases with age. The amplitude and half duration of the medium-duration AHP also decrease with age, whereas the incidence of post-spike afterdepolarization (ADP) drastically increases during this period. Inoue et al ${ }^{39)}$ demonstrated that the maximal repetitive firing rate at steady state is high in MMNs throughout postnatal development, but there is an increase with age in the percentage of MMNs with constant or slightly decrementing firing patterns, as well as an increase in MMNs showing an extra spike. These characteristics result in an initial high-frequency discharge in MMNs even when excitatory inputs are weak. The initial high firing might allow for rapid elevation of the jaw-closing muscle activity that is sufficient to chew food, since insertion of a single extra spike in a low-frequency firing can lead to prolonged tension enhancement generated by the muscle fibers innervated by the related motoneurons ${ }^{40)}$. These developmental changes in the intrinsic properties of MMNs might contribute to the functional transformation from suckling to mastication.

\section{Conclusion}

Neonatal rat pups show suckling behavior to obtain milk immediately after birth. Teeth start to erupt at around P7, immature chewing emerges at around P12, and adult chewing is attained 


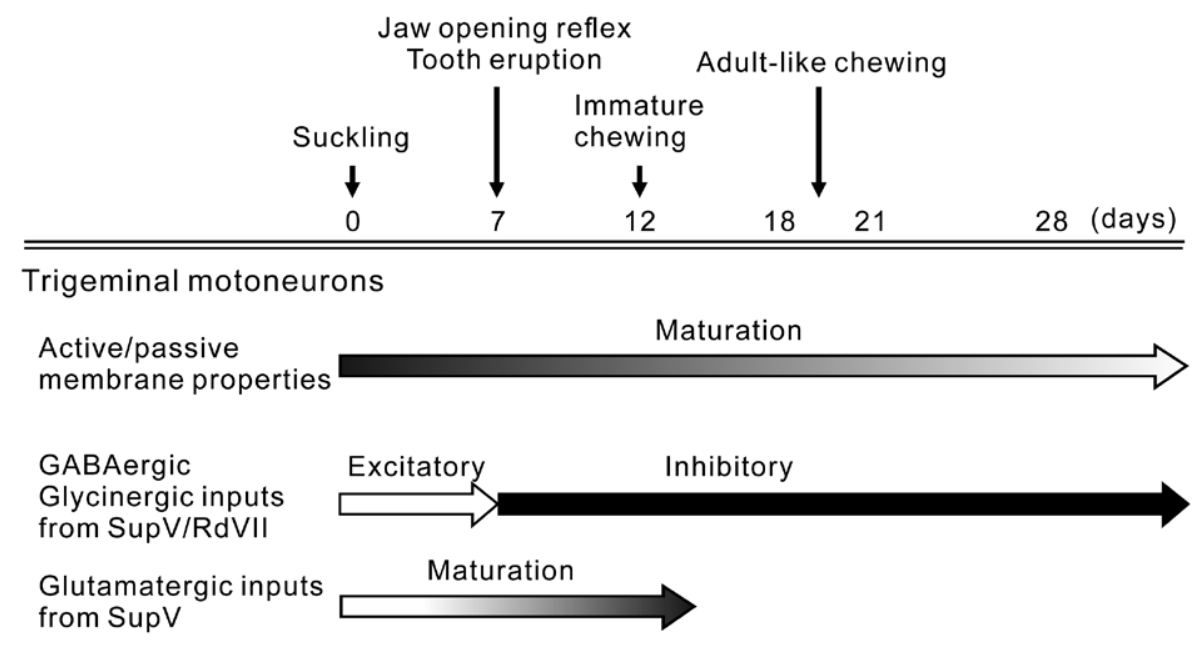

Fig. 4. Timeline for the early postnatal development of orofacial functions and physiological properties of trigeminal motoneurons in rats.

by P18-2128) (Fig. 4). Findings overall indicate that synaptic and intrinsic properties of the TMNs drastically change during the first two postnatal weeks, preceding the initiation of immature chewing. It is likely that these developmental changes play an important role in the proper transition from suckling to mastication by preparing the ability to precisely control masticatory force prior to the initiation of mastication. Herein, we reviewed those studies providing a better cell-based understanding of the neural mechanisms underlying jaw movement during suckling and mastication.

\section{Acknowledgments}

The studies in this article were approved by the Institutional Animal Research Committee of Showa University in accordance with Japanese Government Law No.105. The authors wish to thank all members of the Department of Oral Physiology of Showa University School of Dentistry and all authors of the relevant studies. This work was supported by the Nakatomi Foundation, the MEXT-Supported Program for the Strategic Research Foundation at Private Universities 2014-2018, and the JSPS KAKENHI (JP16K11488).

\section{Conflict of interest disclosure}

The authors have no conflict of interest to declare.

\section{References}

1) Weijs WA, Dantuma R. Functional anatomy of the masticatory apparatus in the rabbit. Neth J Zool. 1980;31:99-147.

2) Inoue $\mathrm{T}$, Kato $\mathrm{T}$, Masuda $\mathrm{Y}$, et al. Modifications of masticatory behavior after trigeminal deafferentation in the rabbit. Exp Brain Res. 1989;74:579-591.

3) Morimoto $\mathrm{T}$, Inoue $\mathrm{T}$, Masuda $\mathrm{Y}$, et al. Sensory components facilitating jaw-closing muscle activities in the rabbit. Exp Brain Res. 1989;76:424-440.

4) Nakamura Y, Katakura N. Generation of masticatory rhythm in the brainstem. Neurosci Res. 1995;23:1-19.

5) Lund JP. Mastication and its control by the brain stem. Crit Rev Oral Biol Med. 1991;2:33-64.

6) Inoue $\mathrm{T}$, Masuda Y, Nagashima $\mathrm{T}$, et al. Properties of rhythmically active reticular neurons around the trigeminal 
motor nucleus during fictive mastication in the rat. Neurosci Res. 1992;14:275-294.

7) Inoue T, Chandler SH, Goldberg LJ. Neuropharmacological mechanisms underlying rhythmical discharge in trigeminal interneurons during fictive mastication. $J$ Neurophysiol. 1994;71:2061-2073.

8) Donga R, Lund JP, Veilleux D. An electrophysiological study of trigeminal commissural interneurons in the anaesthetized rabbit. Brain Res. 1990;515:351-354.

9) Lund JP, Kolta A, Westberg KG, et al. Brainstem mechanisms underlying feeding behaviors. Curr Opin Neurobiol. 1998;8:718-724.

10) Yoshida A, Taki I, Chang Z, et al. Corticofugal projections to trigeminal motoneurons innervating antagonistic jaw muscles in rats as demonstrated by anterograde and retrograde tract tracing. J Comp Neurol. 2009;514:368-386.

11) Hatanaka $\mathrm{N}$, Tokuno $\mathrm{H}$, Nambu $\mathrm{A}$, et al. Input-output organization of jaw movement-related areas in monkey frontal cortex. J Comp Neurol. 2005;492:401-425.

12) Notsu K, Tsumori T, Yokota S, et al. Posterior lateral hypothalamic axon terminals are in contact with trigeminal premotor neurons in the parvicellular reticular formation of the rat medulla oblongata. Brain Res. 2008;1244:71-81.

13) Yasui $Y$, Tsumori $T$, Oka $T$, et al. Amygdaloid axon terminals are in contact with trigeminal premotor neurons in the parvicellular reticular formation of the rat medulla oblongata. Brain Res. 2004;1016:129-134.

14) Mizuno N, Yasui Y, Nomura S, et al. A light and electron microscopic study of premotor neurons for the trigeminal motor nucleus. J Comp Neurol. 1983;215:290-298.

15) Travers JB, Norgren R. Afferent projections to the oral motor nuclei in the rat. J Comp Neurol. 1983;220:280-298.

16) Turman J Jr, Chandler SH. Immunohistochemical evidence for GABA and glycine-containing trigeminal premotoneurons in the guinea pig. Synapse. 1994;18:7-20.

17) Turman JE Jr, Chandler SH. Immunohistochemical localization of glutamate and glutaminase in guinea pig trigeminal premotoneurons. Brain Res. 1994;634:49-61.

18) Takamatsu J, Inoue T, Tsuruoka $\mathrm{M}$, et al. Involvement of reticular neurons located dorsal to the facial nucleus in activation of the jaw-closing muscle in rats. Brain Res. 2005;1055:93-102.

19) Iriki A, Nozaki S, Nakamura Y. Feeding behavior in mammals: corticobulbar projection is reorganized during conversion from sucking to chewing. Brain Res Dev Brain Res. 1988;44:189-196.

20) Kolta A, Westberg KG, Lund JP. Identification of brainstem interneurons projecting to the trigeminal motor nucleus and adjacent structures in the rabbit. J Chem Neuroanat. 2000;19:175-195.

21) Li YQ, Takada M, Kaneko T, et al. Premotor neurons for trigeminal motor nucleus neurons innervating the jawclosing and jaw-opening muscles: differential distribution in the lower brainstem of the rat. J Comp Neurol. 1995;356:563-579.

22) Yamamoto M, Moritani M, Chang Z, et al. The somatotopic organization of trigeminal premotoneurons in the cat brainstem. Brain Res. 2007;1149:111-117.

23) Nakamura $\mathrm{S}$, Inoue $\mathrm{T}$, Nakajima $\mathrm{K}$, et al. Synaptic transmission from the supratrigeminal region to jaw-closing and jaw-opening motoneurons in developing rats. $J$ Neurophysiol. 2008;100:1885-1896.

24) Nonaka M, Nishimura A, Nakamura S, et al. Convergent pre-motoneuronal inputs to single trigeminal motoneurons. J Dent Res. 2012;91:888-893.

25) Gemba-Nishimura A, Inoue T, Nakamura S, et al. Properties of synaptic transmission from the reticular formation dorsal to the facial nucleus to trigeminal motoneurons during early postnatal development in rats. Neuroscience. 2010;166:1008-1022.

26) Nakamura S, Nakayama K, Mochizuki A, et al. Electrophysiological and morphological properties of rat supratrigeminal premotor neurons targeting the trigeminal motor nucleus. J Neurophysiol. 2014;111:1770-1782.

27) Nagoya K, Nakamura S, Ikeda K, et al. Distinctive features of Phox2b-expressing neurons in the rat reticular formation dorsal to the trigeminal motor nucleus. Neuroscience. 2017;358:211-226.

28) Westneat MW, Hall WG. Ontogeny of feeding motor patterns in infant rats: an electromyographic analysis of 
suckling and chewing. Behav Neurosci. 1992;106:539-554.

29) Mizuno N, Konishi A, Sato M. Localization of masticatory motoneurons in the cat and rat by means of retrograde axonal transport of horseradish peroxidase. J Comp Neurol. 1975;164:105-115.

30) Uemura-Sumi M, Manabe Y, Matsushima R, et al. Correlation of the main peripheral branches of the facial nerve with the cytoarchitectonic subdivisions of the facial nucleus in the guinea pig. Anat Embryol (Berl). 1986;174:161-166.

31) Miyata H, Sugiura T, Wada N, et al. Morphological changes in the masseter muscle and its motoneurons during postnatal development. Anat Rec. 1996;244:520-528.

32) Matsuda K, Nakamura S, Nonaka M, et al. Premotoneuronal inputs to early developing trigeminal motoneurons. J Oral Biosci. 2017;59:96-103.

33) Bae YC, Nakamura T, Ihn HJ, et al. Distribution pattern of inhibitory and excitatory synapses in the dendritic tree of single masseter alpha-motoneurons in the cat. J Comp Neurol. 1999;414:454-468.

34) Shigenaga Y, Moritani M, Oh SJ, et al. The distribution of inhibitory and excitatory synapses on single, reconstructed jaw-opening motoneurons in the cat. Neuroscience. 2005;133:507-518.

35) Nagata S, Nakamura S, Nakayama K, et al. Postnatal changes in glutamatergic inputs of jaw-closing motoneuron dendrites. Brain Res Bull. 2016;127:47-55.

36) Major G, Polsky A, Denk W, et al. Spatiotemporally graded NMDA spike/plateau potentials in basal dendrites of neocortical pyramidal neurons. J Neurophysiol. 2008;99:2584-2601.

37) Schiller J, Major G, Koester HJ, et al. NMDA spikes in basal dendrites of cortical pyramidal neurons. Nature. 2000;404:285-289.

38) Yamaoka A, Inoue $\mathrm{T}$, Hironaka S, et al. Postnatal changes in electrophysiological properties of rat jaw-closing motoneurons. Showa Univ J Med Sci. 2005;17:71-79.

39) Inoue $\mathrm{T}$, Yamaoka A, Hironaka S, et al. Postnatal changes in the repetitive firing properties of rat jaw-closing motoneurons. J Jpn Soc Stomatognath Funct. 2013;19:137-144.

40) Burke RE, Rudomin P, Zajac FE 3rd. Catch property in single mammalian motor units. Science. 1970;168:122-124.

[Received July 4, 2017 : Accepted July 31, 2017] 